\title{
Las competencias digitales como vehículo de la cultura organizacional universitaria
}

\section{Digital skills as a vehicle for university organizational culture}

\author{
José Daniel Barquero Cabrero. ESERP Business \& Law School, España. \\ jd.barquero@eserp.com \\ $[\mathrm{CV}] \bigcirc \mathrm{G}$ \\ Mercedes Cancelo Sanmartín. Universidad de Málaga, España. \\ Inv. Invitada en la Universidad del Valle de México (México) \\ cancelo@uma.es \\ [CV] \\ Leticia Rodríguez Segura. Universidad del Valle de México. \\ Leticia.rodriguez@uvmnet.edu \\ $[\underline{\mathrm{CV}}] \odot \mathrm{C}$
}

Investigación financiada por Universidad de Málaga y Observatorio Eserp-Abertis.

Cómo citar este artículo / Referencia normalizada

Barquero Cabrero, J. D., Cancelo Sanmartín, M. y Rodríguez Segura, L. (2021). Las competencias digitales como vehículo de la cultura organizacional universitaria. Revista Latina de Comunicación Social, 79, 17-33. https://www.doi.org/10.4185/RLCS-2021-1495

\section{RESUMEN}

La constante digitalización de la sociedad y por ende de las organizaciones ha supuesto una redefinición de los procesos comunicativos. Estos cambios han penetrado con avidez en la gestión de la comunicación interna, provocando la redefinición de la vehiculización de la cultura organizacional. En un entorno cambiante la gestión de las organizaciones supone implementar nuevas estrategias de comunicación que aúnen la globalización y las nuevas tecnologías (Wichels, 2014). En esta línea, hemos analizado el valor del uso de las competencias digitales como elemento de traslación de la cultura organizacional en el ámbito universitario. El estudio se ha realizado a partir del método de estudio de caso tomando bajo la premisa de conveniencia cuatro universidades del ámbito público y privado pertenecientes a España y México. Se han analizado en el caso de España: la Universidad de Málaga y ESERP Business \& Law School; y en México la Universidad Autónoma de Tamaulipas y la Universidad del Valle de México. Con el fin de contrastar la penetración de cultura organizacional de forma simétrica en el modelo universitario público y privado en el panorama Iberoamericano. Las técnicas de estudio utilizadas se han basado en la observación directa no participante, aplicación de encuestas a público interno (empleados), realización de entrevistas en profundidad a los responsables de la gestión comunicativa y análisis de los recursos digitales utilizados en la comunicación interna. Como avance de resultados se puede apreciar la aplicación de dos modelos diferentes con claras influencias continentales. La instrumentalización de la cultura organizacional en las universidades estudiadas está enfocada de 
forma diferente en función de su origen público o privado, siendo más efectiva en su penetración y por lo tanto, en su identificación en el caso de entes privados.

El desafío de la cultura organizacional en el ámbito universitario radica en la necesidad de acrecentar el valor identitario a través de un ideario real y colectivo que se viva y plasme en la gestión cotidiana.

PALABRAS CLAVE: Cultura organizacional; competencias digitales; identidad; universidad; México; España.

\begin{abstract}
The constant digitalization of society and therefore of organizations has meant a redefinition of communication processes. These changes have eagerly penetrated the management of internal communication, causing the redefinition of the vehiculization of organizational culture. In a changing environment, the management of organizations means implementing new communication strategies that combine globalization and new technologies (Wichels, 2014). In this line, we have analyzed the value of the use of digital competencies as an element of translation of the organizational culture in the university environment. The study was carried out based on the case study method taking four public and private universities belonging to Spain and Mexico under the premise of convenience. They have been analyzed in the case of Spain: the University of Malaga and ESERP Business \& Law School; and in Mexico the Autonomous University of Tamaulipas and the University of the Valley of Mexico. In order to contrast the penetration of organizational culture symmetrically in the public and private university model in the Latin American landscape. The study techniques used have been based on direct non-participant observation, application of internal public surveys (employees), conducting in-depth interviews with those responsible for communicative management and analysis of digital resources used in internal communication. As an advance of results you can see the application of two different models with clear continental influences. The instrumentalization of the organizational culture in the universities studied is focused differently depending on their public or private origin, being more effective in their penetration and therefore, in their identification in the case of private entities. The challenge of organizational culture in the university field lies in the need to increase identity value through a real and collective ideology that is lived and embodied in everyday management.
\end{abstract}

KEYWORDS: Organizational culture; digital skills; identity; University; Mexico; Spain.

\title{
CONTENIDOS
}

1. Introducción. 2. Objetivos. 3. Metodología. 4. Resultados. 5. Conclusiones y discusión. 6. Referencias.

\section{Introducción}

La digitalización de las organizaciones ha supuesto no solo una modificación en los procesos de trabajo sino también en las estructuras comunicativas hacia el interior de los entes (Marta-Lazo et al, 2020; Mayoral et al, 2019). El desarrollo tecnológico abarca todas la dinámicas organizacionales y potencia de esta forma la implementación y el desarrollo de lo que conocemos como competencias digitales (Schena et al, 2018; Blanco Alfonso et al, 2019). En el estudio que se plantea se ha interrelacionado la implementación de las competencias digitales con la cultura organizacional, debido a que las competencias del ámbito digital son un instrumento de transmisión de valores culturales e identitarios en cada organización (Vaquerizo Domínguez, 2019; Blasco Magraner y Botella Nicolás, 2020). 
Sobre la base del espíritu propugnado por Niño González y Linares Herrera (2020) el punto de partida ha sido la existencia a priori de una visión de identidad organizacional, y la transmisión de la misma a través de la estrategia de comunicación interna y sus instrumentos.

El modelo organizacional que se aborda en esta investigación es el universitario. Se analizan centros universitarios públicos y privados con el fin de obtener una visión paritaria de los procesos de comunicación y de la cultura organizacional.

La universidad es una organización que por sus características estructurales y de operación debe adoptar nuevas metas y mecanismos flexibles para su evolución y aceptación por el entorno (GinésMora, 2004). Esta visión organizacional deposita en el personal universitario la responsabilidad de gestión del liderazgo desde una perspectiva humana, la generación de procesos de cambio que se adecuen a través de la cultura organizacional y una estrategia nítida que propicie los cambios que demanda la sociedad en su conjunto (Montañez-García, 2017).

La comunicación interna, desde una perspectiva amplia, siempre se ha considerado una construcción organizacional compleja y con altas dificultades, tal y como lo han señalado Del Pozo (1997), Barth (2004), Andrade (2005), Fernández (2007), Rodríguez (2007), Pizzolante (2009), entre otros.

El reto de la comunicación interna se acrecienta cuando nos circunscribimos al ámbito universitario. La universidad es un ente que por su naturaleza requiere un ejercicio de alta responsabilidad social, que ofrezca respuestas a la sociedad con una constante adaptación de sus procesos y mecanismos de trabajo. Esta génesis organizacional eleva potencialmente la dificultad de su gestión en comunicación interna como han señalado diferentes investigaciones realizadas por Aguirre et al. (2012), GinésMora (2004 y 2014), Imbernón (2008), Lau (2009), Llano (2003), Pena-Vega (2009), Aristizábal y Hernández (2014), entre otros. Todos estos análisis previos observan los procesos comunicativos en el seno universitario desde diferentes perspectivas con un nexo común: la dificultad de integrar a los miembros bajo una secuencia cultural única. A su vez, todos esos estudios coinciden en la necesidad de construir una visión de universidad que sea fortalecida desde dentro a través de la integración de su público interno, con especial atención a sus miembros permanentes, es decir, el personal.

\section{Objetivos}

La cultura organizacional es "el (la) lente con el que debemos mirar las posibilidades de cambio en las instituciones de educación superior" (Montañez-García, 2017, p. 55). La cultura universitaria es por lo tanto la respuesta a los comportamientos y desarrollos organizacionales, que se integran en la vida organizacional generando modelos de comportamiento y experiencias compartidas que permiten la inclusión y la aceptación (Rifkin, 2000).

En nuestro estudio definiremos la cultura organizacional como "un patrón complejo de normas, actitudes, creencias, valores, ceremonias, tradiciones y mitos que están profundamente integrados en el interior de la organización" (Barth, 2004, p. 160).

Las competencias digitales son un instrumento clave en la gestión organizacional, y en el caso de la universidad son una piedra angular en el presente y futuro de la organización social (Feijoo Fernández y Guerrero, 2018; Bello van der Ree y Morales Lozano, 2019). Su papel de generadoras de conocimiento obliga a estos entes a adaptar la totalidad de sus procesos a una visión digital. Es importante señalar que las competencias de forma genérica se definen como "la capacidad para el desempeño con éxito de funciones y papeles en un ámbito" (De la Orden Hoz, 2011, p. 47). En 
concreto, las competencias digitales se definen como "el uso crítico y seguro de las Tecnologías de la Sociedad de la Información para el trabajo, el tiempo libre y la comunicación; apoyándose en habilidades como el uso de ordenadores para recuperar, evaluar, almacenar, producir, presentar e intercambiar información, y para comunicar y participar en redes de colaboración a través de Internet" (DOL394, 2006) en unos principios también detallados por González Gascón (2017).

Los estudios previos realizados que se enfocan en el análisis de las competencias digitales en la universidad sólo abordan el papel de las competencias en el ámbito de la formación y el desarrollo docente (García-Valcárcel et al, 2010), (Páez y Carlo, 2012), (Arias, Torres y Yáñez, 2014), (MartínAntoranz et al, 2019). Por lo que respecta a la comunicación interna y la cultura organizacional, en la universidad existen diferentes estudios de análisis en el ámbito Iberoamericano (Aristizábal y Hernández, 2014), (Montañez-García, 2017), (Fernández Beltrán, 2016), (Martínez, 2018) que ponen de manifiesto la complejidad en el trazado de la comunicación interna en una realidad universitaria que no siempre ha contemplado la comunicación interna como un motor de gestión organizacional.

Con base en una profusa revisión bibliográfica, esta investigación aúna dos elementos hasta ahora no relacionados entre sí: las competencias digitales y la cultura organizacional. Estas piezas son clave para la articulación de un esfuerzo endógeno que conducirá a las universidades hacia un modelo de gestión más ágil para enfrentarse a los cambios y retos que la sociedad le demanda (MontañezGarcía, 2017).

\section{Metodología}

Se presenta un estudio que tiene como objetivo determinar qué valor posee el uso de las competencias digitales como elemento transmisor de la cultura organizacional en la universidad. El análisis de este escenario se deriva del hecho de que la implementación de las habilidades digitales se ha convertido en un valor en alza en las organizaciones, sobre todo en aquellas orientadas a la enseñanza superior.

Usar las competencias digitales como elemento transmisor de la comunicación interna posiciona a las universidades. En la actualidad, estas organizaciones no solo se valoran por sus procesos productivos, sino también por sus procesos de comunicación. Por lo tanto, la digitalización de su comunicación facilita la instrumentalización de su crecimiento interno.

Derivado de este objetivo central, se pretende conocer el grado de penetración de la cultura organizacional en los empleados universitarios con el fin de determinar el nivel de identificación con la organización.

Las competencias digitales se han conformado a partir de las señaladas por la International Society for Technology in Education (ISTE) en su documento NETS for Teachers (2008), y se combinan con las competencias digitales presentes en los análisis de gestión organizacional propuestas por Prensky (2001) y la Comisión Europea (2018) en la gestión profesional del ámbito educativo. Estas competencias se ordenan en tres grupos: TIC (Tecnologías de la Información y la comunicación), TAC (Tecnologías del aprendizaje y el conocimiento) y TEP (Tecnologías del empoderamiento y la participación), según su orientación sea funcional, educativa o participativa.

Las competencias digitales valoradas en este estudio son: Conocimiento digital, Gestión de la información, Comunicación digital, Trabajo en red, Visión estratégica y Orientación organizacional. La selección de las mismas responde al mismo criterio funcionalista organizacional de la muestra escogida. 
Para aglutinar los elementos que componen la cultura en la universidad, se ha utilizado el modelo de arbusto propuesto por Rodríguez (2007). En el citado modelo las hojas del árbol son aquellos elementos que son visibles de la cultura como los comportamientos, ceremonias, lenguaje, instalaciones, historia, simbología y lemas o eslóganes. Las raíces supondrán los aspectos no visibles a simple vista que se compondrían de valores, creencias y normas organizacionales.

El estudio, de carácter mixto, se ha elaborado desde una perspectiva descriptiva y explicativa, a partir del método de estudio de caso tomando bajo la premisa de conveniencia cuatro universidades del ámbito público y privado pertenecientes a España y México.

Se ha analizado en el caso español la Universidad de Málaga y ESERP Business \& Law School, esta última universidad privada con presencia en Madrid, Barcelona y Palma de Mallorca. En México la selección se ha compuesto de la Universidad Autónoma de Tamaulipas y el ente privado Universidad del Valle de México con presencia en 18 estados de los 31 que integran a México. La bifocalidad de la naturaleza organizacional se basa en el fin de contrastar de forma simétrica y comparada el modelo universitario público y privado en el panorama Iberoamericano.

En la selección de la muestra, se ha determinado previamente la clasificación de público interno de las universidades a partir del Modelo de los públicos de universidades e instituciones culturales de Noguero (1982). De esta estratificación se ha seleccionado el grupo de personal (docente y administrativo). Esto implica que la muestra se ha orientado al personal de las organizaciones universitarias incluyendo a directivos, personal de administración/servicios y docentes/investigadores. Se ha delimitado dentro del público interno al empleado organizacional, excluyendo a los estudiantes, debido a que la orientación del estudio se centra en los miembros constitutivos de la organización. Es decir, aquellos que realizan las actividades que hacen posible el funcionamiento de la misma. En añadidura, se considera que ya existe un amplio acervo de estudios que han determinado la identidad y el desarrollo digital de las universidades en función de una muestra integrada exclusivamente por estudiantes. Por lo tanto, la selección de muestra que se presenta en este análisis establece una perspectiva nueva y complementaria a las existentes.

La muestra se ha compuesto de un total de 301 miembros de las 4 universidades. Teniendo en cuenta el porcentaje de paridad de hombres y mujeres en cada uno de los centros de educación superior. El reparto de análisis de muestra se compone de 77 miembros en el caso de la Universidad de Málaga, 77 participantes en el caso de ESERP Business \& Law School, 77 integrantes de la Universidad Autónoma de Tamaulipas y 70 componentes de la plantilla de la Universidad del Valle de México. La edad de los miembros no se ha tomado como un factor relevante y se ha hecho partícipe del estudio a todos aquellos empleados que tuviesen una vinculación superior a 1 año sin importar su permanencia indefinida o temporal a la organización. Esto último en razón de que la variabilidad contractual del vínculo no exime de la necesidad de conformar la identidad organizacional o el uso de las competencias digitales para la integración de la misma.

Las técnicas de estudio utilizadas se han basado en la observación directa no participante, aplicación de encuestas a personal, realización de entrevistas en profundidad a los responsables de la gestión comunicativa de cada universidad y análisis descriptivo de los recursos digitales utilizados en la comunicación interna.

La aplicación de encuestas se ha realizado a un total de 297 miembros de las 4 universidades, se ha eliminado de la muestra inicial a los responsables de comunicación interna de cada universidad por poseer una visión estructuradora sobre el objeto de estudio. 
La encuesta se ha conformado de 19 preguntas divididas en tres bloques diferenciados: conocimiento de la identidad organizacional, comunicación interna y construcción de la cultura organizacional a través de las competencias digitales y, valoración de la identidad compartida. Para la confección de la encuesta se partió de una adaptación derivada del cuestionario de análisis de comunicación interna universitaria de Fernández Beltrán (2016), generando un modelo mixto integrado por respuestas en base a opciones descriptivas del accionar comunicativo y respuestas de medición escala Likert.

La observación directa no participante se realizó en las cuatro universidades con el total de la muestra, siendo plasmada en un diario de notas de campo. Caracterizando el instrumento por una realización estructurada basada en el acotamiento del objeto de estudio y en la implementación de aspectos medibles en la observación. La presencia del investigador fue abierta por lo tanto los miembros organizacionales eran conscientes de la investigación. Por último, la generación fue natural ya que no se propició ningún escenario creado, sino que se analizó en real y cotidiano de cada universidad.

Se practicaron 4 entrevistas en profundidad a los responsables de comunicación interna de cada universidad participante. Las entrevistas en profundidad presentan un mapa amplio de respuestas razonadas de las cuales se obtiene información pormenorizada sobre aspectos como motivaciones, valores y experiencias del entrevistado (Wimmer y Dominick, 1996).

La entrevista en profundidad (Grinnell1, 1997) estuvo estructurada en base a un cuestionario de 60 preguntas divididas en cuatro bloques: identificación del entrevistado y funciones organizacionales, creación y desarrollo de planes de comunicación interna, análisis de la generación de cultura organizacional y, uso de las competencias digitales como vehículos de la cultura organizacional.

Por último, se realizó un análisis de los recursos digitales utilizados por parte de las cuatro universidades estudiadas. Para ello se estableció una tabla de análisis de elementos de comunicación digital (Arango, 2013), compuesta por 15 aspectos descriptivos orientados a determinar su usabilidad, transmisión visual de la identidad y reflejo de la misión-visión organizacional.

El uso de las cuatro técnicas de estudio presentadas consigue una triangulación de datos de diferente naturaleza y fuentes. Por lo tanto los hallazgos de investigación presentados avalan con mayor certeza a las conclusiones que aporte este estudio (Hernández Sampieri et al., 2006).

\section{Resultados}

Inicialmente es relevante mencionar que los resultados de la presente investigación no se han presentado sino hasta contar con el estudio completo y con resultados concluyentes. Se han evitado por la tanto las publicaciones parciales de cualquier segmento de la investigación.

La aplicación de las encuestas al personal de las cuatro universidades participantes se realizó de forma aleatoria e intencional. Las premisas buscadas eran que participasen miembros con un vínculo superior a un año, paridad en el género de la suma total de encuestados, representación del sector directivo en cada organización, representación del personal docente/investigador y representación de los miembros de administración/servicios.

Para la elección de la muestra se tuvo en cuenta la diseminación de los campus universitarios de las cuatro universidades. Las cuatro universidades poseen un número muy dispar de campus o sedes. Por ello, a fin de determinar en qué sedes o campus se implementaría la investigación, se buscó 
establecer un criterio común aplicable a cada centro universitario. Se establecía, por tanto, la elección de los campus en función de un ratio superior al 50\% de las sedes de cada universidad, siendo las seleccionadas aquellas que respondían al mayor número de empleados y a su importancia estratégica en los planes de desarrollo universitario. Este criterio determino una correlación entre unidades de análisis y unidades existentes, buscando siempre un promedio por encima del $50 \%$ en cada caso.

Se presenta cuadro aclaratorio de la elección de las sedes:

Tabla 1. Número Campus por Universidad

\begin{tabular}{|c|c|c|c|c|}
\hline Universidad & $\mathrm{N}^{\mathrm{o}}$ sedes & $\begin{array}{c}\text { Nombre de campus/Sedes } \\
\text { (a) }\end{array}$ & $\begin{array}{l}\text { Muestra escogida } \\
\text { (b) }\end{array}$ & Ratio \\
\hline $\begin{array}{l}\text { Universidad de } \\
\text { Málaga }\end{array}$ & 2 & $\begin{array}{l}\text { Teatinos (Málaga) } \\
\text { El Ejido (Málaga) }\end{array}$ & $\begin{array}{l}\text { Teatinos } \\
\text { El Ejido }\end{array}$ & $2: 2$ \\
\hline $\begin{array}{l}\text { Eserp Business \& } \\
\text { Law School }\end{array}$ & 3 & $\begin{array}{l}\text { Barcelona } \\
\text { Madrid } \\
\text { Palma de Mallorca }\end{array}$ & $\begin{array}{l}\text { Barcelona } \\
\text { Madrid }\end{array}$ & $2: 3$ \\
\hline $\begin{array}{l}\text { Universidad } \\
\text { Autónoma de } \\
\text { Tamaulipas }\end{array}$ & 8 & $\begin{array}{l}\text { Cd. Victoria (Tamaulipas) } \\
\text { Mante (Tamaulipas) } \\
\text { Tampico (Tamaulipas) } \\
\text { Reynosa (Tamaulipas) } \\
\text { Matamoros (Tamaulipas) } \\
\text { Nuevo Laredo (Tamaulipas) } \\
\text { Valle Hermoso (Tamaulipas) } \\
\text { Rio Bravo (Tamaulipas) }\end{array}$ & $\begin{array}{l}\text { Cd. Victoria } \\
\text { Tampico } \\
\text { Reynosa } \\
\text { Matamoros } \\
\text { Nuevo Laredo }\end{array}$ & $5: 8$ \\
\hline $\begin{array}{ll}\text { Universidad del } \\
\text { Valle de México }\end{array}$ & 36 & $\begin{array}{l}\text { Chapultepec (Estado de } \\
\text { México) } \\
\text { Coyoacan Roma (Estado de } \\
\text { México) } \\
\text { San Rafael (Estado de México) } \\
\text { Centro Santa Fe (Estado de } \\
\text { México) } \\
\text { Centro Marina (Estado de } \\
\text { México) } \\
\text { Hispano (Estado de México) } \\
\text { Lago Gudalupe (Estado de } \\
\text { México) } \\
\text { Lomas Verdes (Estado de } \\
\text { México) } \\
\text { Textcoco (Estado de México) } \\
\text { Toluca (Estado de México) } \\
\text { Tlalpan (Estado de México) } \\
\text { Aguascalientes } \\
\text { (Aguascalientes) } \\
\text { Mexicali (Baja California) } \\
\text { Tuxtla (Chiapas) } \\
\text { Chihuahua (Chihuahua) } \\
\text { Saltillo (Coahuila) } \\
\text { Torreon (Coahuila) } \\
\text { Guadalajara Sur (Jalisco) } \\
\text { Guadalajara Zapopan (Jalisco) } \\
\text { Cuernava (Morelos) } \\
\text { Monterrey Cumbres (Nuevo } \\
\text { León) } \\
\text { Monterrey Norte (Nuevo León) } \\
\text { Puebla (Puebla) } \\
\text { Queretaro (Querétaro) } \\
\text { San Luis Potosí (San Luis } \\
\text { Potosí) }\end{array}$ & $\begin{array}{l}\text { Chapultepec } \\
\text { Coyoacan Roma } \\
\text { San Rafael } \\
\text { Centro Santa Fe } \\
\text { Lomas Verdes } \\
\text { Toluca } \\
\text { Tlalpan } \\
\text { Aguascalientes } \\
\text { Mexicali } \\
\text { Guadalajara Sur } \\
\text { Guadalajara } \\
\text { Zapopan } \\
\text { Cuernava } \\
\text { Mty. Cumbres } \\
\text { Mty. Norte } \\
\text { Queretaro } \\
\text { Villahermosa } \\
\text { Cd. Victoria } \\
\text { Reynosa } \\
\text { Tampico } \\
\text { Veracruz }\end{array}$ & $21: 36$ \\
\hline
\end{tabular}




\begin{tabular}{|l|l|l|l|}
\hline & Hermosillo (Sonora) & & \\
& Centro Hermosillo (Sonora) & & \\
& Nogales (Sonora) & & \\
& Villahermosa (Tabasco) & & \\
& Cd. Victoria (Tamaulipas) & & \\
& Reynosa (Tamaulipas) & & \\
& Tampico (Tamaulipas) & & $30: 49$ \\
\hline & Veracruz (Veracruz) & & \\
\hline Rérida (Yucatán) & \\
\hline
\end{tabular}

Fuente: Elaboración propia

La encuesta se presentó a través de un formato de Google forms, explicando previamente las características y el fin del estudio. Asimismo, se garantizó el anonimato a los participantes así como se generó un espacio libre para la realización de apreciaciones por parte de los encuestados.

El periodo de aplicación de las encuestas fue en el caso de la Universidad de Málaga durante el mes de noviembre del 2019, en la Universidad Autónoma de Tamaulipas en el mes de diciembre de 2019, en ESERP Business \& Law School y en la Universidad del Valle de México durante los primeros días del mes de enero de 2020.

Los resultados se procesaron a través del programa de análisis estadístico SPSS 20.0 para Mac (IBM inc.), en la semana posterior a la aplicación de cada grupo de encuestas y finalmente se realizó un cruce de resultados de las cuatro universidades en la tercera semana del mes de enero.

La observación directa no participante se consideró un instrumento fundamental en el trabajo de campo porque proporciona un análisis de características de comportamiento organizacional. Es un punto de partida investigador básico para observar un conjunto de sujetos que se desarrollan bajo tipos de conductas concretas que responden a diferentes grados de estructuración organizacional (Fernández, 1995). La observación se realizó de forma estructurada con un diario de notas de campo en el cual estaban identificados los objetivos de estudio, el campo de acción y la guía de observación. El grupo de observación en cada universidad integró a 16 miembros de cada organización en los cuales existió paridad de género, puestos a diferentes niveles jerárquicos y perfil profesional. El ejercicio observacional y recopilación de datos se llevó a cabo en los meses de octubre y noviembre de 2019 en las 4 universidades participantes.

Las entrevistas en profundidad se practicaron en base a un cuestionario de 60 preguntas, dando la posibilidad de ampliar y derivar la respuesta por parte del entrevistado. Se buscó en la aplicación de este instrumento determinar la visión estratégica organizacional referente al desarrollo de la comunicación interna y la cultura en cada universidad. Vinculando la aplicación de competencias digitales al mapa organizacional como vehículo cultural del ente universitario.

Las entrevistas se realizaron a través de entrevista en persona y envío por email, con el fin de permitir a los entrevistados reflexionar sobre las cuestiones presentadas. Así como en algunas preguntas realizadas, recabar datos que sustentasen sus respuestas. La técnica se aplicó durante los meses de diciembre de 2019 y los primeros días del mes de enero de 2020 a las cuatro universidades integrantes del estudio.

Por último se realizó un análisis descriptivo de los recursos digitales que se utilizan por parte del personal de cada organización universitaria. La determinación de estos recursos se realizó a partir de 
los obtenidos en la observación directa no participante y la aplicación de las encuestas al personal universitario. Es importante determinar que se estableció la premisa de que para ser considerados recursos digitales debían ser identificados como tales por parte de los integrantes de cada universidad, así como utilizados por ellos mismos en sus actividades cotidianas antes de la realización este estudio. Finalmente se cruzó la lista de elementos analizados con aquellos que los responsables de comunicación ofrecieron como herramientas de comunicación digital desde el punto de vista de la dirección estratégica. Los parámetros evaluados fueron su usabilidad, transmisión visual de la identidad y reflejo de la misión-visión organizacional a través de una plantilla de análisis. La recopilación y análisis de datos se desarrolló en las 4 universidades durante los meses de diciembre de 2019 y los primeros días de enero de 2020.

Es importante señalar que el proceso metodológico es un ejercicio que plantea al investigador una constante reflexión así como en ocasiones permite una redefinición de los instrumentos utilizados en el trabajo de campo. En esta investigación se planteó un proceso empírico que fue evolucionando y matizándose durante la implementación de la misma. Estas redefiniciones instrumentales fueron realizadas con el fin de asegurar el desarrollo estratégico y la consecución de los objetivos del estudio. En este sentido, la observación directa brindo la posibilidad de redefinir elementos planteados a priori en la encuesta al personal universitario, así como matizar preguntas elaboradas para la entrevista en profundidad a los responsables de comunicación interna de las universidades participantes.

Tras las entrevistas realizadas a los responsables de comunicación de las universidades analizadas se puede establecer que la planeación de la comunicación es asumida por cada uno de los responsables. En el caso de las universidades públicas estudiadas dicho responsable asume también de forma íntegra la dirección de la comunicación interna. En el caso de la Universidad del Valle de México y Eserp Business \& Law School, ambas privadas, el diseño de la comunicación interna se comparte con la dirección de recursos humanos. A pesar de esta bicefalidad, las mayores competencias en materia de toma de decisiones y diseño de estrategias se toman bajo la dirección de comunicación. Los responsables de comunicación de las cuatro universidades poseen un perfil adecuado para el desarrollo de sus competencias, todos ellos tienen estudios en comunicación. Aunque en el caso de la Universidad de Málaga el responsable de comunicación pertenece a la rama de periodismo, y en de Eserp Business \& Law School está más vinculado con el marketing. Estos perfiles profesionales inciden en la posterior aplicación de la planificación de la comunicación, así como la utilización de elementos para la transmisión de la cultura organizacional.

Las entrevistas aplicadas permiten extraer información acerca de una visión organizacional en la que se establece la existencia de competencias digitales que refuerzan la transmisión del ser organizacional, así como la construcción de una cultura conjunta. Desde la dirección de comunicación de las cuatro universidades se estima que hay un estímulo suficiente para que exista un sentir común entre el personal universitario. Asimismo, los responsables de comunicación coinciden respecto a que, bajo la lógica temporal, aquellos miembros que son fijos comparten un mayor sentimiento de pertenencia y por lo tanto contribuyen decisivamente al crecimiento y transmisión de la cultura organizacional.

En las entrevistas realizadas a los responsables de comunicación de la Universidad de Málaga y de Eserp Business \& Law School se plasma la visión de que las competencias digitales fundamentales que están presenten en la transmisión de la cultura organizacional son el conocimiento digital, la gestión de la información, comunicación digital, el trabajo en red, La visión estratégica y la orientación organizacional. Estas competencias según se deriva de las dos entrevistas, se plasman a través del uso de los recursos digitales presentes en las dos universidades. 
En el caso de la Universidad Autónoma de Tamaulipas y la Universidad del Valle de México las competencias digitales se integran en la comunicación de forma natural ya que diseñan los contenidos digitales desde una perspectiva comunicativa clara, tomando el ideario de la organización como el eje de construcción de cada elemento digital. En ambas universidades mexicanas, la cultura organizacional supone un aspecto vivo y constante que se revisa en cada plan de comunicación y se enriquece a través de la retroalimentación con el personal universitario. En la observación directa no participante se establecieron claras diferencias en función de los países del caso de estudio. En las universidades mexicanas, Universidad Autónoma de Tamaulipas y Universidad del Valle de México, los valores organizacionales están presentes en la cotidianidad del personal. Los directivos se relacionan con el resto de los miembros utilizando las premisas que identifican el Ser de la Universidad tanto desde el punto de vista visual como en los aspectos constitudinarios culturalmente. Existe un accionar institucional basado en la ejemplificación de la dirección hacia el resto de puestos y grupos jerárquicos. Entre los docentes se comparte una visión de grupo y por lo tanto identidad que se ve reforzada por los elementos contenidos en las competencias digitales. Se observa la utilización de las competencias digitales a través de las acciones comunes, las competencias habituales son el conocimiento digital, la gestión de la información, la comunicación digital, el trabajo en red, la visión estratégica, así como la predominancia de las acciones orientadas a los objetivos organizacionales.

En el caso de Ésera Business \& Law School existe una visión integral de los procesos de trabajo en los que se promueve la utilización de las competencias digitales en las que se contiene a su vez la esencia cultural de la organización. Se denota un esfuerzo constante por parte de los directivos en mantener acciones que refuercen y referencien el uso de las competencias digitales.

En el caso de la Universidad de Málaga se observa cierta confusión en el uso de las competencias digitales en función del rango de edad y nivel de pertenencia a la organización. Si bien no se planteó la edad como un rango importante en el diseño instrumental, en este caso se demostró una correlación entre edad-competencias-cultura organizacional. Los miembros más jóvenes sin diferencia en el nivel jerárquico, en vinculación permanente o no, y en el rol organizacional (directivo, docente/investigador y administración/servicios) desarrollan una labor cotidiana integrada por las competencias digitales, en las cuales se identifica claramente el hilo cultural que comunica la visión y misión organizacional con su actividad diaria. Por lo tanto, en este caso específico la cultura organizacional se transmite de una forma eficaz y constante entre los miembros más jóvenes del personal de la Universidad de Málaga.

Las encuestas realizadas mantienen una clara correlación con la observación directa no participante y una coincidencia variable con las entrevistas en profundidad a los responsables de la comunicación organizacional de cada universidad. El análisis derivado de las encuestas demuestra en primera instancia que la cultura organizacional en la Universidad de Málaga no es un elemento que se observe por parte del personal de forma simétrica, existiendo ciertas disidencias con respecto a la intención de los responsables de comunicación.

\section{Tabla 2. Resultados de Cultura Organizacional}

\begin{tabular}{|l|c|c|}
\hline $\begin{array}{c}\text { ¿Conoces la cultura organizacional de tu universidad? Se entiende por } \\
\text { cultura organizacional el conjunto de valores, normas, conductas que } \\
\text { unifican los comportamientos de los empleados y que ayudan a trabajar en } \\
\text { una misma dirección. }\end{array}$ & Si & No \\
\hline ESERP & $100 \%$ & $0 \%$ \\
\hline UMA & $67 \%$ & $33 \%$ \\
\hline
\end{tabular}


RLCS, Revista Latina de Comunicación Social, 79, 17-33

[Investigación] 10.4185/RLCS-2021-1495| ISSN 1138-5820| Año 2021

\begin{tabular}{|l|c|c|}
\hline UVM & $93 \%$ & $7 \%$ \\
\hline UAT & $75 \%$ & $25 \%$ \\
\hline
\end{tabular}

Fuente: Elaboración propia

Respecto al grado de identificación de los valores culturales en las universidades estudiadas, existen ciertas variaciones con respecto a las pretensiones de los estrategas de comunicación interna.

Tabla 3. Resultados de Cultura Organizacional

\begin{tabular}{|l|c|c|}
\hline $\begin{array}{c}\text { En caso afirmativo, ila } \\
\text { compartes? }\end{array}$ & $\mathrm{Si}$ & No \\
\hline ESERP & $100 \%$ & $0 \%$ \\
\hline UMA & $90 \%$ & $10 \%$ \\
\hline UVM & $85 \%$ & $15 \%$ \\
\hline UAT & $95 \%$ & $5 \%$ \\
\hline
\end{tabular}

Fuente: Elaboración propia

Existe entre el personal de las cuatro universidades una visión diferente resto a la implementación de las competencias digitales vinculadas a la cultura organizacional.

Tabla 4. Resultados de Cultura Organizacional-Competencias digitales

\begin{tabular}{|l|c|c|}
\hline $\begin{array}{l}\text { Crees que en la cultura organizacional de tu universidad está } \\
\text { presente en las competencias digitales y que estas ofrecen un } \\
\text { valor extra a la comunicación interna? }\end{array}$ & $\mathrm{Si}$ & No \\
\hline ESERP & $100 \%$ & $0 \%$ \\
\hline UMA & $74 \%$ & $26 \%$ \\
\hline UVM & $87 \%$ & $13 \%$ \\
\hline UAT & $100 \%$ & $0 \%$ \\
\hline
\end{tabular}

Fuente: Elaboración propia

Respecto al uso de las competencias digitales en el caso de la Universidad Autónoma de Tamaulipas el $65 \%$ de los encuestados determinan que son fundamentales para el desarrollo institucional. Un $15 \%$ del personal asume las competencias digitales como importantes en el accionar institucional y un $10 \%$ considera poco relevante la implementación de competencias digitales y finalmente un $10 \%$ lo considera irrelevante y un $0 \%$ del todo irrelevantes.

En el caso de la Universidad del Valle de México el $76 \%$ de los encuestados coinciden en que las competencias digitales son fundamentales para el desarrollo institucional, un $18 \%$ del personal encuestado las considera importantes, un $6 \%$ del personal piensa que las competencias digitales son poco relevantes, finalmente, nadie de los encuestados las encuentra irrelevantes y del todo irrelevantes.

En las encuestas realizadas en Eserp Business \& Law School un 86\% considera las competencias digitales fundamentales para el desarrollo institucional, un 14\% las concibe como importantes. Dejando desiertas las opciones de irrelevantes y del todo irrelevantes.

En la Universidad de Málaga un 55\% considera a las competencias digitales fundamentales para el desarrollo institucional, un $25 \%$ determina que las competencias digitales son importantes, un $17 \%$ las observa como irrelevantes y un $3 \%$ del todo irrelevantes. 
Respecto a los recursos digitales que son utilizados por el personal encuestado de las 4 universidades analizadas tenemos los siguientes resultados:

Tabla 5. Resultados de Cultura Organizacional-Competencias digitales

\begin{tabular}{|l|l|l|l|l|l|l|l|}
\hline $\begin{array}{c}\text { En mi organización la } \\
\text { comunicación interna } \\
\text { digital se aplica a través } \\
\text { de... (marque todas las } \\
\text { que apliquen) }\end{array}$ & Intranet & $\begin{array}{c}\text { Web } \\
\text { pública }\end{array}$ & email & $\begin{array}{c}\text { Redes } \\
\text { Sociale } \\
\mathrm{s}\end{array}$ & $\begin{array}{c}\text { Campus } \\
\text { Virtual }\end{array}$ & $\begin{array}{c}\text { Tablon } \\
\text { es } \\
\text { digitale } \\
\text { s }\end{array}$ & $\begin{array}{c}\text { Grupos de } \\
\text { WhatsApp }\end{array}$ \\
\hline ESERP & $\mathrm{X}$ & $\mathrm{X}$ & $\mathrm{X}$ & $\mathrm{X}$ & $\mathrm{X}$ & & \\
\hline UMA & $\mathrm{X}$ & $\mathrm{X}$ & $\mathrm{X}$ & & $\mathrm{X}$ & $\mathrm{X}$ & \\
\hline UVM & $\mathrm{X}$ & & $\mathrm{X}$ & $\mathrm{X}$ & $\mathrm{X}$ & & \\
\hline UAT & $\mathrm{X}$ & $\mathrm{X}$ & $\mathrm{X}$ & $\mathrm{X}$ & $\mathrm{X}$ & $\mathrm{X}$ & \\
\hline
\end{tabular}

Fuente: Elaboración propia

El análisis de los recursos digitales confirma que existe una constante y adecuada usabilidad, transmisión visual de la identidad y reflejo de la misión-visión organizacional. En este sentido es necesario aclarar que en las entrevistas a los responsables de comunicación interna, el número de recursos digitales declarados era superior a los que el personal utiliza y manifiesta como existentes. Cabe dicha mención dado que, como se indicó en el apartado metodológico, se han considerado para este estudio analítico sólo aquellos recursos digitales que son reconocidos y utilizados por el personal universitario.

\section{Conclusiones y discusión}

Existe en las universidades analizadas desde la dirección de comunicación una conciencia clara de que la comunicación interna es vital para las organizaciones. También existe una coincidencia general en observar la cultura como un elemento vivo y vital para la sanidad organizacional. Así como el apoyo indiscutible que suponen las competencias digitales para la transmisión de esta cultura compartida. En el diseño y en las intenciones organizacionales las directrices son claras pero en la transacción a la cotidianidad se pierde esta concatenación de elementos.

En primer lugar, en las universidades mexicanas existe un alto sentido de pertenencia institucional, que se vincula directamente con el conocimiento compartido de la cultura en cada una de las universidades. En el caso español existe una diferencia notable entre la universidad pública y las privadas. En el caso de la Universidad de Málaga la identidad organizacional no es totalmente reconocida y por ende la cultura no es un elemento altamente compartido. En contraste, en Eserp Business \& Law School, la identidad organizacional está presente en la mayoría de los miembros de la universidad y la cultura organizacional es un ámbito compartido casi por la totalidad. Esta diferencia, manifiesta en el caso español, el nivel de penetración de la comunicación interna y sus planes estratégicos entre el personal de la universidad pública respecto a la privada es altamente variable. Existe por lo tanto una instrumentalización de la cultura organizacional con marcadas diferencias a los ojos del personal universitario. Es más efectiva en el caso de España el modelo de penetración de la identidad organizacional y en consecuencia de la cultura en los entes privados que en los públicos.

La cultura organizacional se presenta como un elemento de construcción y crecimiento constante en todos los casos analizados, pero se plantean amplias diferencias en los matices y en la aceptación. En 
el caso de las universidades mexicanas se pone de evidencia que la cultura forma parte del hacer cotidiano de la organización. Los directivos universitarios se implican con su ejemplo diario en la translación de los valores que los identifican y a su vez son reconocidos e implementados por el resto de los miembros de cada universidad.

En el caso español, la universidad privada se predispone a ejercitar de forma diaria aquellos valores que las diferencian del resto de organizaciones, en un alto porcentaje la penetración en la plantilla es eficaz. Ya que no encuentra resistencia a la búsqueda de una cultura compartida, debido principalmente a la percepción de que integran un modelo empresarial. En la universidad pública existe una marcada diferencia en la capacidad de aceptar una cultura compartida por parte del personal. Los miembros más jóvenes son permeables a los valores culturales mientras que los más antiguos se muestran reticentes a entender la importancia de este elemento organizacional. En base a que no existe una cultura institucional realmente reconocida en las organizaciones universitarias de cáliz público.

Las competencias digitales son observadas casi en su totalidad como elementos necesarios en la transmisión de la cultura organizacional. Así lo demuestra la identificación y el uso de los recursos digitales en las universidades. Aunque desde la visión del plan de comunicación interna existen más recursos no identificados por los miembros de las universidades. Lo cual pone de relevancia la necesidad de implementar un plan de acción específica en la promoción y utilización de esos recursos desaprovechados.

En consecuencia, las competencias digitales sí suponen un vehículo eficaz para la cultura organizacional. Aunque es necesario acrecentar la identidad organizacional en las universidades públicas españolas. Existe una herencia negativa que se superpone al trabajo comunicativo y que lastra la cultura organizacional desvirtuándola. Siendo está reconocida como un concepto ideal y no realmente materializado en el devenir organizacional.

Es necesario afrontar el desafío de la cultura organizacional en el ámbito universitario, buscando acrecentar el valor de identidad compartida a través de un ideario que sea reconocido y compartido por todos los miembros de la organización.

\section{Referencias}

Aguirre, R., De Pelekais, C. \& Paz, A. (2012). Responsabilidad social: Compromiso u obligación universitaria. Telos, 14(1), 11-20. ISSN 1317-0570.

Andrade, H. (2005). Comunicación organizacional interna: proceso, disciplina y técnica. Madrid: Netbiblio.

Arango-Forero, G. (2013). Comunicación digital: una propuesta de análisis desde el pensamiento complejo. Palabra Clave 16 (3), 673-697. https://www.redalyc.org/pdf/649/64930924002.pdf

Arias Oliva, M., Torres Coronas, T., \& Yáñez Luna, J-C. (2014). El desarrollo de competencias digitales en la educación superior. Revista Historia y comunicación social, $n^{o}$ Especial, (19), 355366. https://doi.org/10.5209/rev_HICS.2014.v19.44963

Aristizábal Giraldo, M. E. \& Hernández García, D. (2014). Estudio comparativo de la gestión de la comunicación interna en universidades públicas con acreditación de alta calidad en Antioquía. 
https://aprendeenlinea.udea.edu.co/revistas/index.php/folios/article/view/326293

Barth, R. S. (2004). Culture in question. En Jossey-Bass Publishers (Ed.), The Jossey-Bass Reader on educational leadership (2da. e159-168). San Francisco.

Bello van der Ree, M. E. y Morales Lozano, J. A. (2019). Competencias claves de los estudiantes universitarios para el uso de las TIC. Revista de Comunicación de la SEECI, 50, 43-72. https://doi.org/10.15198/seeci.2019.50.43-72

Blanco Alfonso, I., García Galera, C. y Tejedor Calvo, S. (2019). El impacto de las fake news en la investigación en Ciencias Sociales. Revisión bibliográfica sistematizada. Historia y Comunicación Social, 24(2), 449-469. https://doi.org/10.5209/hics.66290

Blasco Magraner, J. S. y Botella Nicolás, A. M. (2020). Aprendizaje por proyectos en la clase de bachillerato: la opereta La Corte del Faraón. Revista de Comunicación de la SEECI, 51, 1-15. http://doi.org/10.15198/seeci.2020.51.1-15

CA: John Wiley \& Sons. Comisión Europea. Nuevas medidas para impulsar las competencias clave, las capacidades digitales y la dimensión europea de la educación. 2018. http://europa.eu/rapid/press-release IP-18-102 es.pdf.

De La Orden Hoz, A. (2011). El problema de las competencias en la educación general. Bordón, 63, (1),47-61. https://recyt.fecyt.es/index.php/BORDON/article/view/28904

Del Pozo, M. (1997). Cultura empresarial y comunicación interna. Madrid: Fragua.

DO, Diario Oficial de las Comunidades Europeas, L394 (2006). Recomendación del Parlamento Europeo y del Consejo de 18 de diciembre de 2006 sobre las Competencias Clave para el Aprendizaje Permanente. content/ES/TXT/PDF/?uri=CELEX:32006H0962\&from=ES

http://eur-lex.europa.eu/legal-

Feijoo Fernández, B. y Guerrero, J. J. (2018). La investigación de mercados en redes sociales: conoce-tu consumidor por lo que publica en Facebook y Twitter. Análisis de caso de ADT Security Services en Chile. Vivat Academia, Revista de Comunicación. 144, 19-35. http://doi.org/10.15178/va.2018.144.19-35

Fernández Ríos, M. (1995). Análisis y descripción de puestos de trabajo. Teoría, métodos y ejercicios. Madrid: Ediciones Díaz de Santos.

Fernández, F. (2007). La gestión de la nueva comunicación interna. Análisis de la aplicación de las tecnologías de la información en los procesos de comunicación interna de las universidades de la Comunidad Valenciana. EBSCO. http://tesisenred.net/handle/10803/10464

Ginés-Mora, J. (2004). La necesidad del cambio educativo para la sociedad del conocimiento. Revista Iberoamericana de Educación, $\quad 35, \quad$ 13-37. https://doi.org/10.35362/rie350874 
Ginés-Mora, J. (2014). La Universidad: Un futuro incierto. Conferencia Magistral ofrecida en la Universidad de Puerto Rico, Recinto de Río Piedras, en la celebración del 50ta aniversario del Departamento de Estudios Graduados de la Facultad de Educación, el 31 de marzo de 2014.

Grinnell, R. M. (1997). Social work research \& evaluation: Quantitative and qualitative approaches. Itaca: E. E. Peacock Publishers.

González-Gascón, E. (2017). El trabajo colaborativo como herramienta para el desarrollo personal. Una experiencia en una asignatura de marketing. Revista de Ciencias de la Comunicación e Información, 22(2), 75-88. http://doi.org/10.35742/rcci.2017.22(2).75-88

Hernández Sampieri, R., Fernández Collado, C. \& Baptista Lucio, P. (2006). Metodología de la investigación. México: McGraw Hill.

Imbernón, F. (2008). Los retos de la universidad del futuro. Recuperado de http://univnova.org/documentos/6.pdf recuperado (10/12/2019).

Llano, A. (2003). Repensar la universidad: La universidad ante lo nuevo. Pamplona, Madrid: Ediciones Internacionales http://www.univforum.org/sites/default/files/RepensarUniversidad_es.pdf

Lau, R. (2009). Características ideales de la Universidad futura. Reencuentro, 56, 130-133. https://reencuentro.xoc.uam.mx/index.php/reencuentro/article/view/731

Marta-Lazo, C., Rodríguez Rodríguez, J. M. y Peñalva, S. (2020). Competencias digitales en periodismo. Revisión sistemática de la literatura científica sobre nuevos perfiles profesionales del periodista. Revista Latina de Comunicación Social, 75, 53-68. http://doi.org/10.4185/RLCS-2020$\underline{1416}$

Martín-Antoranz, P., Cabezuelo-Lorenzo, F. y Bartolomé-Muñoz-de-Luna, A. (2019). Competencias y formación ética de los profesionales de la comunicación corporativa en contextos interculturales. Revista de Ciencias de la Comunicación e Información, 24(1), 59-72. http://doi.org/10.35742/rcci.2019.24(1)59-72

Martínez Arellano, N. A. (2018). Gestión de la comunicación interna en la Universidad Pública. El caso de la Facultad de Ciencias Administrativas y Sociales de la UABC. Diagnóstico y plan de intervención. Revista Razón y Palabra, 100, (22), 837-855. https://archivos.revistarazonypalabra.org/index.php/ryp/article/view/1138

Mayoral, J., Parratt, S. y Morata, M. (2019). Desinformación, manipulación y credibilidad periodísticas: una perspectiva histórica. Historia y Comunicación Social, 24(2), 395-409. https://doi.org/10.5209/hics.66267

Montañez-García, A. (2017). Liderazgo, cultura organizacional y cambio en la universidad. Cuadernos de investigación en la educación, 32, 51-62. http://cie.uprrp.edu/cuaderno/2018/01/18/liderazgo-cultura-organizacional-y-cambio-en-launiversidad/

NETS for Teachers (2008). https://people.umass.edu/pelliott/reflections/netst.html 
Niño González, J. I. y Linares Herrera, M. P. (2020). Investigar: Acción impostergable. Bibliotecas. Anales de Investigación, 16(1), 5-6. http://www.bnjm.cu/revistaanales/Revista\%20Anales\%20Vol\%2016\%20No.1\%202020/01-Vol-16-N-1-2020-Editorial.pdf

Noguero, A. (1982). Relaciones Públicas e industria de la persuasión: análisis gnoseológico y situacional. Madrid: Eunibar.

Páez, R. \& Carlo, S. (2012). Aproximación docimológica a la evaluación de competencias digitales y didácticas de profesores universitarios. Revista Iberoamericana de Evaluación Educativa, 5, (1), 284-288. http://hdl.handle.net/10486/661710

Pena-Vega, A. (2009). El porvenir de la universidad: Consideraciones sobre el futuro en un contexto mundial. Reencuentro, 54, https://reencuentro.xoc.uam.mx/index.php/reencuentro/article/view/685

Pizzolante, I. (2009). El desafío de modelar. Comunicación estratégica para el fortalecimiento institucional. http://www.venamcham.org/demo/eventos/perspectivas_soc/italo_pizzolante.pdf

Prensky, M. (2001). Digital natives, digital immigrants. On the horizon. MCB University Press, 9, 16

Rifkin, J. (2000). La era del acceso: La revolución de la nueva economía. Barcelona: Paidós.

Rodríguez, A. (2007). Propuesta de modelos culturales orientados a la gerencia del cambio. Acimed, 15(4). http://scielo.sld.cu/pdf/aci/v15n4/aci05407.pdf

Schena, J., Besalú, R. y Singla Casellas, C. (2018). Valoraciones actualizadas de las competencias profesionales en la práctica laboral de los periodistas españoles. Revista Latina de Comunicación Social, 73, 531-555. http://doi.org/10.4185/RLCS-2018-1268

Vaquerizo Domínguez, E. (2019). Medios étnicos, adaptación al ecosistema digital y usos de la diáspora migrante. Vivat Academia, Revista de Comunicación. 149, 127-151. http://doi.org/10.15178/va.2019.149.127-151

Wichels, S. (2014). Nuevos desafíos en Relaciones Públicas 2.0: La creciente influencia de las plataformas de online review en Turismo. Revista Internacional de Relaciones Públicas, 7, (IV), 197-216. http://dx.doi.org/10.5783/RIRP-7-2014-12-197-216

Wimmer, R. y Dominick, J. (1996). La investigación científica de los medios de comunicación. Barcelona: Bosch.

\section{AUTORES:}

\section{José Daniel Barquero Cabrero}

ESERP Business \& Law School- Observatorio ESERP-Abertis. El Prof. Titular y Catedrático José Daniel Barquero Cabrero es doctor en el área de las Ciencias Económicas y Sociales por: Universitat Internacional de Catalunya (Barcelona); Universidad Camilo José Cela (Madrid), Universidad Autónoma de Coahuila (México) e interuniversitariamente por las Universidades de Málaga, Huelva, Cádiz y Sevilla. También es Economista colegiado núm. 13.049. Ha sido galardonado por sus 
aportes al mundo académico con el título de doctor honoris causa por universidades de América, Europa, Asia y África y por el Gobierno de España, Ministerio de Asuntos Exteriores y de Cooperación, con la Encomienda de la Orden del Mérito Civil, otorgada por S.M el Rey, así como por la Fundación de Fomento Europeo con la Cruz Europea de Oro. Es Miembro del Patronato de ESERP Business \& Law School y Profesor-Catedrático en Economía y Empresa en La UOLS-Open University La Salle. Asesora a empresas e instituciones financieras y bancarias españolas. Presidente honorífico del Consejo Superior Europeo de Doctores, académico de la Real Academia de Ciencias Económicas y Financieras de España; académico de la Real Academia de Doctores, académico de la Academia Iberoamericana de Doctores, instituciones todas de las que forman parte numerosos premios Nobel, con los que ha compartido sus investigaciones. Ha trabajado en EEUU con el pionero mundial de las Relaciones Públicas, el Profesor Doctor Edward L. Bernays Freud, asesor de presidentes de Estados Unidos y de la Casa Blanca y, en el Reino Unido, con el Profesor Doctor Sir Sam Black, asesor de S.M. la Reina de Inglaterra y empresas líderes. Sus libros han sido publicados en siete países distintos: España (McGraw-Hill, Deusto, Planeta, Universidad de Barcelona); Reino Unido (Staffordshire University); Federación Rusa (Ediciones Dielo); México (Editorial Trillo); Azerbaiyán y Estados Unidos (Editorial McGrawHill), Portugal (Porto Editora).

jd.barquero@eserp.com

Orcid ID: https://orcid.org/0000-0003-3927-0529

Google Scholar: https://scholar.google.com/citations?hl=es\&user=p vGd90AAAAJ

\section{Mercedes Cancelo Sanmartín.}

Universidad de Málaga, España. Doctora en Ciencias de la Comunicación por la Universidad de Málaga, Posgrado en Gestión de Políticas Públicas por la Universidad Nacional de a Plata (Argentina) y Estudios de Doctorado en Criminología con orientación a victimología, nuevas tecnologías y riesgo institucional, por la Universidad de Granada. Pionera en el estudio de la comunicación en Fuerzas y Cuerpos de seguridad del Estado. Sus líneas de investigación son la comunicación organizacional con especial atención a la comunicación institucional, opinión pública, incidencia de nuevas tecnologías en el ámbito público y el papel de los grupos vulnerables en las redes sociales. Investigadora Invitada en la Universidad del Valle de México, investigadora integrada en el Observatorio ESERP- Abertis y miembro Investigador en European Center for Social Science Reserch (ECSSR).

cancelo@uma.es

Orcid ID: https://orcid.org/0000-0002-8595-5505

\section{Leticia Rodríguez Segura}

Universidad del valle de México. Directora Nacional de Innovación e Investigación Educativa en la Universidad del Valle de México. Licenciada en Psicología por la UNAM, Maestra en Psicología Educativa por la UNAM y Maestra en Ciencias de la Educación Universidad del Valle de México. Miembro del Sistema Mexicano de Investigación en Psicología (SMIP) y del Grupo Académico Nacional para la Reforma Integral de la Educación Básica en México.

leticia.rodriguez@uvmnet.edu

Orcid ID: https://orcid.org/0000-0002-2356-4170

Google Scholar: https://scholar.google.com/citations?hl=es\&user=Cx3rAO8AAAAJ 